\title{
Association between Hasford Scoring System and Hematologic Response in Chronic and Accelerated Phase of Chronic Myelocytic Leukemia Patient with Imatinib for Three Months
}

\author{
Andy Purnomo, Ugroseno Yudho Bintoro, Made Putra Sedana, Ami Ashariati \\ Hematology Oncology Medic Sub-specialist Program, Department of Internal Medicine, Faculty of Medicine, \\ Universitas Airlangga/Dr. Soetomo General Hospital, Surabaya, Indonesia
}

Background: Hasford score is a scoring system which was made in interferon treatment era to assess chronic myelocytic leukemia (CML) prognosis. Complete hematologic response (CHR) is the milestone of prognosis evaluation. $\mathrm{CHR}$ achievement will significantly increase survival. Imatinib is a revolutionized treatment that change the prognosis of CML. With the advent of Imatinib, lessened the prognostic impact of the Hasford score to predict prognosis.

Materials and Methods: An observational analytic with prospective cohort study conducted in oncology outward division Dr. Soetomo hospital Surabaya, from July until October 2018. Hasford score determined in 32 patients at the beginning of the study, given imatinib and followed up regularly for 3 months to know the hematologic response. Data were analyzed using Fisher exact test which was considered significant if $p<0.05$.

Results: Median age was 39 years old, male $37.5 \%$ and female $62.5 \%$, the median spleen was $18 \mathrm{~cm}$, median hemoglobin was $9.1 \mathrm{~g} / \mathrm{dL}$, median leukocyte was $180 \times 10^{9} / \mathrm{L}$, median thrombocyte was $645 \times 10^{9} / \mathrm{L}$, median eosinophil was $2.9 \%$, median basophil was $4.6 \%$, median myeloblast was $6 \%$. Hasford score showed $3.1 \%$ in low risk, $25 \%$ in intermediate risk and $71.9 \%$ in high risk. As much as $78.1 \%$ complete hematologic response was found in patient, and $21.9 \%$ was incomplete.

Conclusion: There was no association between Hasford scoring system and hematologic response in chronic and accelerated phase of chronic myelocytic leukemia patient with imatinib for three month. Hasford score had no impact in hematologic response with imatinib.

Keywords: Hasford score, hematologic response, CML, imatinib

\section{Introduction}

Chronic myelocytic leukemia (CML) is a myeloproliferative disease marked by leukocytosis, bone marrow hyperplasia and splenomegaly with the appearance of Philadelphia chromosome as its signature which inside there was a breakpoint cluster region protein (BCR)-Abelson murine leukemia viral oncogene homolog $(\mathrm{ABL})$ gene fusion that

Date of submission: January 7, 2019

Last Revised: February 26, 2019

Accepted for publication: March 4, 2019

Corresponding Author:

Andy Purnomo

Hematology oncology Medic Sub-specialist Program

Department of Internal Medicine

Faculty of Medicine, Universitas Airlangga/Dr. Soetomo General Hospital

Jl. Mayjen Prof. Dr. Moestopo 6-8, Surabaya, 60286, Indonesia

E-mail: andypurnomo.ap@gmail.com

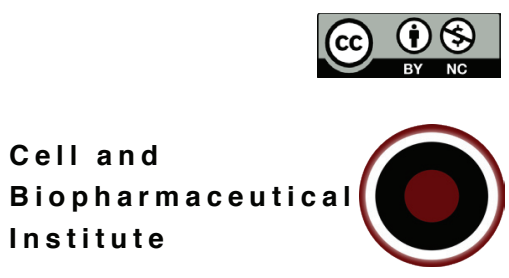


was form because of a reciprocal translocation between the long arm of ABL1 gene of the chromosome 9 with the short arm of BCR gene of the chromosome 22 and written as $\mathrm{t}(9 ; 22)$ ( $\mathrm{q} 34 ; \mathrm{q} 11)$. Philadelphia chromosome was proven to carry a unique fusion gene called BCR-ABL, which was believed to be the main cause of CML in chronic phase.

Around $15 \%$ of all leukemia around the world was CML. CML incidence in some countries varies even when there were not a lot of differentiations between them. Around 4,000-5,000 new cases were diagnose in the USA annually. CML incidence is at 1.6-2.0 cases per 100,000 people each year. In China, it was estimated that $0.39-0.55$ per 100,000 population with the median age of 45-50 years and with the male to female ratio of 1.5:1. In Thailand it was around 0.5 in every 100,000 population with the median age $36-38$ years old and with the male and female ratio of 1.7:1. In Indonesia, CML incidence was at 1.7 per 100,000 residents in the general hospital Dr. Soetomo of Surabaya. There was an increase in incidence where in 2006, there were 58 patients mostly at the age of 30-40 years old, but in 2014 there were $160 \mathrm{CML}$ patients. ${ }^{2}$ Even if CML could occur at all age groups, its incidence increased at every decade of life, it makes the disease to become an adult disease. According to Surveillance, Epidemiology and End Result (SEER), the average age when the diagnosis of the disease was 66 years old, a much higher age than reported on a lot of clinical trial. This disease also appeared more often in male patients that female patients $(2.2: 1.3){ }^{3}$

There were three known important risk factor scores in CML, which were Sokal, Hasford and European Treatment and Outcome Study (EUTOS) score, which was made in different time and use to grade the prognosis of CML. ${ }^{4}$ Hasford score originated from a multivariate survival analysis in 981 chronic phase CML patients which were treated in 1983-1994. All of those patients were treated with only a single interferon alpha medicine or combine with other therapy. Where as in Hasford's research low risks were discovered in $40.6 \%$ of the patients, intermediate risks in $44.7 \%$ of patients and high risks in $14.6 \%$ of patients. This score was validated in a separate cohort research on 322 CML patients. ${ }^{5}$ Several other researches about the prognosis score showed the following result. Sinha, stated that Hasford score was a better predictive prognosis indicator compared to Sokal, whereas Hasford score had two extra variables among them were the presentation of eosinophil and Basophil, this was not the case with the other score. The more eosinophil and basophil the worse the prognosis.
Basophil was the source of angiogenesis inflammation process and also a source of fibrogenic molecules such as vascular endothelial growth factor or hepatocyte growth factor. Basophil also contain vasoactive substances such as histamine and cytokine dissolving enzyme, dipeptidylpeptidase IV that cause the mobilization of stem cell and the extra medullar spreading of stem cell and progenitor cell. Basophil also produced autocrine growth factor in CML. ${ }^{6}$ Thomas also stated that Sokal score was no longer the best suited to predict CML patients survival. ${ }^{7}$ Dybko stated that Hasford score was still usefull as a tool to differentiate low and intermediate risks in achieving molecular response where as Sokal and EUTOS did not. ${ }^{8}$ To this day Sokal score was still the main score that was widely use, because of that researches in Hasford score was still need to be conducted.

Hematologic response was an important point to asses a prognosis. This response was evaluated in the first three months. Failure to reach a complete hematologic response in three months is rare and usually signed a bad prognosis. ${ }^{9}$ Complete hematologic response (CHR) was seen in $74 \%$ of patients that received Imatinib treatment. ${ }^{10}$ Bilen stated that CHR also occurred in $100 \%$ who received Imatinib. CHR was seen in $91 \%$ of patients with Imatinib. ${ }^{11}$ CHR was also seen in $90 \%$ of patients with Imatinib. ${ }^{12}$ Hematologic response also occurred in $52 \%$ of blastic crisis phase CML patients that received Imatinib. ${ }^{13}$ Studies on therapeutically hematologic response were very important regarding prognosis and its simple and economical nature to be conducted.

Imatinib was a medicine use in several cancers with positive Philadelphia chromosome $(\mathrm{Ph}+)$ therapy like $\mathrm{CML}$. The five years survival rate for CML patients increased twice from 31\% (before imatinib) in 1993 to 59\% for those who were diagnose on 2003-2009 with imatinib. Annual mortality rate decreased from $10-20 \%$ to about $2 \%$. With introduction of tyrosine kinase inhibitor (e.g., imatinib, nilotinib, dasatinib) into CML therapy lessened the prognostic impact of the prognostic factor. Pretreatment prognostic factor like Hasford lost much of clinical relevance regarding prognosis and to select therapies. ${ }^{14}$ These prognostic scales require revalidation given the dramatic impact of conversion to tyrosine kinase inhibitor treatment. ${ }^{15}$ Based on the reviews and considerations above, the researcher wants to know the association between Hasford score and hematological response in three months of Imatinib treatment. It was hoped that from this research, data can be provided to help 
determine the role of Hasford score toward theurapeutical hematological response in this imatinib therapy era.

\section{Materials and methods}

This research was an analytic, cohort prospective study using primary data conducted in the oncology outward division, Dr. Soetomo Hospital, Surabaya, from July-October 2018. The target populations of this research were CML BCRABL positive patients which had not Imatinib treatment. The inclusion criteria was new male or female patients above the age of 18 years old and agreed to participate in this research which were proven in an informed consent. The exclusion criteria were patients who had liver cirrhosis, chronic hepatitis B or C and sepsis. Meanwhile the drop out citeria were pregnant (amenorrhea with pregnancy test positive), blastic crisis transformation (blast count $>30 \%$ ), patient could not take imatinib for less than 1 week, and loss of treatment monitoring.

The starting dose for Imatinib was at $400 \mathrm{mg}$. If there was a drug side effect grade 3 or 4 , the dose would be reduced to $300 \mathrm{mg}$.

\section{Hasford Scoring System}

The examination of BCR-ABL gene transcript of the CML patients in this research was conducted through real time PCR method using GeneXpert machine. If resulted positive, patient's age, spleen size, and count of hemoglobin, leukocyte, thrombocyte, eosinophil, basophil were recorded. Examination of blood count using Sysmex XN-1000 machine. The examination of the myeloblast count was taken from the peripheral blood smear examined by two examiners, one examiner weas from clinical pathology division, while the other one was from internal medicine division. Researcher conducted an inter-rater agreement between the examiners. Chronic phase of the CML if myeloblast count $<15 \%$ and accelerated phase if myeloblast count $15-30 \%$. Hasford score was calculated using Hasford calculator online, then Hasford score was grouped into three categories, which were low $(\leq 780)$, intermediate (780-1480) and high ( $\geq 1481)$.

\section{Hematologic Response}

The researches subject was given with Imatinib therapy and then reexamined. The reexamination included symptoms, blood test, peripheral blood smear, and spleen size every months for three months to determine the hematologic response of the patients that was grouped to complete hematologic response define by no sign and symptoms of disease with disappearence of palpable splenomegaly, leukocytes $\leq 10 \times 10^{9} / \mathrm{L}$, thrombocytes $\leq 450 \times 10^{\%} / \mathrm{L}$ and no immature cells such as myelocytes, promyelocites, and blasts in peripheral blood smear, otherwise was incomplete hematologic response.

\section{Statistical Analysis}

Hasford score were ordinal data. Hematologic response were nominal data. The data was non parametric and presented as a median. All of the data from the research was tabulated and analyze using the SPSS software version 18 to calculate the significance value statistically. Statistical analysis was performed using fisher exact test, with significance level was set at $p<0.05$.

\section{Results}

\section{Characteristic of CML Patients}

At the end of the research there were 32 subjects. The researcher subject dominated by female, young age with massive splenomegaly, anemia, severe leukocytosis, thrombocytosis, and basophila. Myeloblast markly increased in blood smear examination. Detail characteristic of the CML patients was shown in Table 1.

Table1. Characteristic of the CML patients.

\begin{tabular}{lc}
\hline \multicolumn{1}{c}{ Characteristic (n=32) } & Median (Range) \\
\hline Gender & $12(37.5)$ \\
$\quad$ Male, $\mathrm{n}(\%)$ & $20(62.5)$ \\
\hline Female, $\mathrm{n}(\%)$ & $39(18-63)$ \\
\hline Age (years old) & $18(0-30)$ \\
Spleen $(\mathrm{cm})$ & $9.1(5.0-12.3)$ \\
Hb $(\mathrm{g} / \mathrm{dL})$ & $180(1-678)$ \\
Leukocyte $\left(10^{9} / \mathrm{L}\right)$ & $645(61-1355)$ \\
Thrombocyte $\left(10^{9} / \mathrm{L}\right)$ & $2.9(0-11)$ \\
Eosinophil $(\%)$ & $4.6(0-25)$ \\
Basophil $(\%)$ & $6(0-17)$ \\
Myeloblast $(\%)$ &
\end{tabular}




\section{Distribution of Hasford Score in CML Patients}

The Hasford score were consisted of three groups which were low, intermediate and high. Most of the research subjects were classified in the high Hasford score. Hasford score distribution of this study was shown in Table 2.

Table 2. The Hasford score distribution.

\begin{tabular}{lc}
\hline \multicolumn{1}{c}{ Hasford } & n (\%) \\
\hline Low & $1(3.1)$ \\
Intermediate & $8(25)$ \\
High & $23(71.9)$ \\
\hline
\end{tabular}

\section{Distribution of Hematologic Response in CML Patients with Imatinib Treatment}

Hematologic response distribution from the complete and incomplete hematologic response was shown in Table 3. Complete hematologic response occurred in most of the patients.

Table 3. Hematologic response distribution.

\begin{tabular}{lc}
\hline \multicolumn{1}{c}{ Hematologic Response } & n (\%) \\
\hline Incomplete & $7(21.9)$ \\
Complete & $25(78.1)$ \\
\hline
\end{tabular}

\section{Distribution of Hasford Score with Hematologic Response in CML Patients with Imatinib Treatment}

Distribution of low, intermediate, high score and the complete and incomplete hematologic responses showed that all of the low and intermediate Hasford score displayed a complete hematologic response where as in the high Hasford score the majority of the patients also displayed complete hematologic response (Table 4).

Table 4. Distribution of Hasford score with hematologic response.

\begin{tabular}{lccc}
\hline \multirow{2}{*}{ Hasford } & \multicolumn{3}{c}{ Hematologic Response } \\
\cline { 2 - 4 } & $\begin{array}{c}\text { Incomplete } \\
\mathbf{n}(\%)\end{array}$ & $\begin{array}{c}\text { Complete } \\
\mathbf{n}(\%)\end{array}$ & Total \\
\hline Low & 0 & $1(100)$ & 1 \\
Intermediate & 0 & $8(100)$ & 8 \\
High & $7(30.4)$ & $16(69.6)$ & 23 \\
\hline
\end{tabular}

The Statistical Association Test between Hasford Score and Hematologic Response in Chronic and Accelerated Phase CML Patient with Imatinib Treatment

To determine the association test, expectation value was conducted in advance. Since an expectation value of $<5$ reach $50 \%$ of the total cell $(3 / 6)$ then Chi Square test were not up to the conditions, so the Fisher exact test was used. The Fisher exact test results showed $p=0.179$ (statistically significance is $p<0.05)$, then it can be concluded that there were no association between Hasford score with hematologic response in chronic myelocytic leukemia chronic and accelerated phase patients that acquired three months Imatinib therapy. The expectation value of Hasford score with Hematologic Response was shown in Table 5.

Table 5. The expectation value of Hasford score with hematologic response.

\begin{tabular}{lcc}
\hline \multirow{2}{*}{ Hasford } & \multicolumn{2}{c}{ Hematologic Response } \\
\cline { 2 - 3 } & Incomplete & Complete \\
\hline Low & 0.2 & 0.8 \\
Intermediate & 1.8 & 6.3 \\
High & 5 & 18 \\
\hline
\end{tabular}

\section{Discussion}

\section{Characteristic of CML Patient}

At the end of this research, it was discovered that the 32 subjects dominated by female $(62.5 \%)$ with the median age of 39 (18-63) years old. Result from this research was similar with a cohort research conducted in Turkey during 2006-2009, that in 31 research samples were also dominated by female $(61.9 \%)$ with the median age of 48.9 (18-75) years old. ${ }^{16}$ This research was a little bit different with a prospective research in Pakistan that was conducted in 2001-2006 where there were 136 patients dominated by male $(63 \%)$ with the median age $33(12-65)$ years old. ${ }^{17}$ Another retrospective study in Egypt from 2008-2013 that had a much bigger sample which was at 167 patients, was dominated by male $(64.7 \%)$ with the median age of 49 (23-74) years old. ${ }^{18}$ In China, a retrospective study done in 2006-2013, acquired 210 patients dominated by male $(60 \%)$ with the median age of $42.22(6-84)$ years old. ${ }^{19} \mathrm{~A}$ multicenter retrospective study by Hasford in 1983-1994 in 1303 samples discovered a result dominated by male with the median age of 49 years old. ${ }^{5}$ The small sample amount 
in this research was caused by the use of primary data (new patients) with the short research time of four months and two drop outs, where in the other hand, other researches required a minimal time of three years and uses secondary data (medical record). This research acquired a majority of female in the result and this was caused by the demographic factor, hence the amounts of female respondence was more than male. This research had the young age median, similar with the research that was conducted by Usman ${ }^{17}$ which caused CML to become a disease that was not only experience by elderly people (in western country, CML was averagely diagnoses in 66 years old) but people with younger ages could also experience this disease.

In this research, from physical examination, the biggest spleen size with the median of $18(0-30) \mathrm{cm}$ was discovered which reached three times size from the result of Bilen's research with the median of $5.87(0-20) \mathrm{cm}$, Xia's research with the median of $6.73(0-21) \mathrm{cm}$, and Tamer's research with the median of $5(2-14,5) \mathrm{cm}$ also Hasford's research with the median of $3.4(0-30) \mathrm{cm} .{ }^{16,18,19}$ This was caused by the subjects who visited the hospital with severe condition and large tumor burden.

In this research the subjects came to the hospital with anemia, severe leukocytosis and thrombocytosis. From the examination of the marginal blood smear, the median hemoglobin of $9.1 \mathrm{~g} / \mathrm{dL}$, leukocytes of $180 \times 10^{9} / \mathrm{L}$, thrombocytes of $645 \times 10^{9} / \mathrm{L}$, was found that was similar with Xia's research where median hemoglobin of $9.6 \mathrm{~g} /$ $\mathrm{dL}$, leukocytes of $188 \times 10^{9} / \mathrm{L}$, thrombocytes of $363 \times 10^{9} / \mathrm{L}$ was found and in Bilen's research where the percentage of haematocrit at $34.32(23-49) \%$, leukocytes of 141.23 $(13-437) \times 10^{9} / \mathrm{L}$, thrombocytes at $513(134-1571) \times 10^{9} / \mathrm{L}$ was found. It was different with the Hasford's own research where a result of hemoglobin at $12(2-18.2) \mathrm{g} /$ $\mathrm{dL}$, leukocytes at $68(1-571) \times 10^{9} / \mathrm{L}$, thrombocytes at 360 (34-3050)x10\%/L was found. ${ }^{16,19}$ This difference happened because in the western country, most of the patients visited the hospital without symptoms and the disease was found by health care screening test, where in the other hand, in the developing countries including in Indonesia, the patient came to the hospital caused by symptoms.

From the differential count in this research, it was found that the median of eosinophil was $2.9(0-11) \%$, basophil was $4.6(0-25) \%$, this was similar with Yamamoto's research with eosinophil at $2.5(0-13) \%$, basophil at $5(0-25.5) \%$, and Xia's research with eosinophil at 3.5\%, basophil at $4.6 \%$, Bilen's research with eosinophil at 3 (1-8)\%, basophil 2.8
(0-9)\%, Hasford's research with eosinophil $2(0-20) \%$, basophil $3(0-21) \%$, Tamer's research with eosinophil 2 $(0-15) \%$, basophil 4 (1-15)\%. ${ }^{16,18-20}$ Excess basophil was a sign of CML, and this variable was the superiority that Hasford score had compared to Sokal's score in evaluating prognosis.

From the peripheral blood smear examination it was discovered the median of myeloblast at $6(0-17) \%$ which was highest when compare to other researches such as Xia with myeloblast of $1.46 \%$, Hasford with myeloblast at $1(0-$ $15) \%$, Yamamoto with myeloblast at $0(0-14) \%$, and Tamer with myeloblast at $2(0-7) \%{ }^{16,18-20}$ The high myeloblasts showed a bad prognosis which was caused by the subject of this research being in a chronic and accelerated phase where in the other hand in other researches it was only chronic phase.

\section{Distribution of Hasford Score in CML Patient}

In this research it was discovered that the majority of the subjects was classified in high score with the sequence of the low, intermediate and high Hasford score each at 3.1\%, $25 \%$ and $71.9 \%$. It was difference with Kuntegowdanahalli's research which was dominated by the intermediate score with the sequence of $20.2 \%, 62.1 \%, 17.5 \%{ }^{21}$ Yamamoto with the sequence of $41.4 \%, 49.6 \%, 9.0 \%$, Castagnetti with the $38 \%, 62 \%$, 0. Tamer with $38.9 \%, 49.1 \%, 12 \%$., Hasford with $38 \%, 51 \%, 11 \% .{ }^{18,20,22}$ Even in other researches it was dominate by low score such as Xia with $48.1 \%, 43.8 \%$, $8.1 \% .{ }^{19}$ In the western countries the highest score was only at $10-20 \%$. The abundant high scores in this research was caused by the acceleration phase which was also being researches, where as in other researches it was only chronic phase that was being researched.

\section{Hematologic Response Distribution in CML Patients Who Still Acquiring Imatinib Treatment}

In this research the Complete Hematologic Response (CHR) occurred in $78.1 \%$ of the patients. Bilen's research stated that $100 \%$ of the patients achieved CHR in three months, where $80.6 \%$ of patients achieved CHR in one month and 19.4\% achieved CHR within 2 months. ${ }^{16}$ Usman stated that 117 (86\%) CHR patients with 79 patients in between them having low score. ${ }^{17}$ Reksodiputro stated that CHR occurred at $74 \%$ of the patients that received Imatinib treatment. ${ }^{10}$ Roko stated that CHR occurred in 91\% CML patients with Imatinib. ${ }^{11}$ Even hematologic response could also happen in $52 \%$ of CML blastic crisis phase that received Imatinib. ${ }^{13}$ 
This research was similar with other researches where the CHR value was high enough even when the acceleration phase was included in this research. In this research 7 (21.9\%) patients did not achieve CHR because of leukocytes above 10 x 109/L (6 patients) and splenomegaly (1 patient).

\section{Distribution of Hasford Score Toward Hematologic Response in CML Who Still Acquiring Imatinib Treatment}

In this research all of the intermediate and low score $(100 \%)$ reached complete hematologic response where in the other hand $69.6 \%$ of the high score patients also reached complete hematologic response. This showed that complete hematologic response occured in the majority of this score with imatinib treatment. Even in the high score Imatinib also give a good therapy response.

Association between Hasford score with Hematologic response in accelerated and chronic phase of CML who still acquiring Imatinib treatment for three months.

In this research this statistical test result showed $p=0.179$, so it can be concluded that there was no association between Hasford score with Hematologic response. Research by Tamer stated that there was a substantial difference between Hasford scores $(p<0.0001)$ at the therapy failure value whereas the high score value of therapy failure was higher compare to the low score. A lot of researches discussed about the correlation between Hasford score with the survival, cytogenetic and molecular therapy response, but there were very rare for a research to connect between Hasford score and hematologic response. It was difficult to compare the result of this research with other researches because of the rarity of a similar research that has been conducted.

This research was affected by some limitations, one of them is the existence of a disturbing variable of hydroxyurea medicine usage, before the research was conducted, this could affect the Hasford score, also the small amount of sample in this research caused by the lack of new CML patient that fulfilled the conditions of the research and the research's short time.

\section{Conclusion}

There was no association between Hasford score and hematologic response in chronic and accelerated phase of CML patients that received Imatinib treatment for three months. Hasford score had no impact in hematologic reponse with imatinib.

\section{Acknowledgment}

This research did not receive any spesific grant from any funding agency in the public, commercial, or not for profit sector.

\section{References}

1. Jabbour E, Cortes JE, Giles FJ, O' Brien S, Kantarijan HM. Current and emerging treatment option in chronic myeloid leukemia. Cancer. 2007; 109(11): 2171-81.

2. Ugroseno YB. Chronic Myelogenous Leukemia Patogenesis, Diagnosis dan Terapi [Dissertation]. Surabaya: Universitas Airlangga: 2014.

3. Rohrbacher M, Hasford J. Epidemiology of chronic myeloid leukaemia . Best Pract Res Clin Haematol. 2009; 22(3): 295-302.

4. Deininger MW. Chronic myeloid leukemia. In: Greer JP, Arber DA, Glader B, List AF, Means RT Jr, Paraskevas F, et al, e ditors. Wintrobe's Clinical Hematology. 13th ed. Philadelphia: Wolters Kluwer Health Lippincott Williams \& Wilkins; 2014. p.1705-19.

5. Hasford J, Pfirrmann M, Hehlmann R, Allan NC, Baccarani M, Kluin-Nelemans JC. A new prognostic score for survival of patients with chronic myeloid leukemia treated with interferon alfa. J Natl Cancer Inst. 1998; 90(11): 850-8.

6. Valent $\mathrm{P}$, Horny HP. The underestimated role of basophils in $\mathrm{Ph}+$ chronic myeloid leukaemia. Eur J Clin Invest. 2018; 48 (10): e13000. doi: 10.1111/eci.13000.

7. Sinha SK, Sinha S, Mandal PK, Bhattacharyya NK, Pandey A, Gupta P. A comparative study of Hasford score and Sokal index in prognostication of the novo chronic myeloid leukemia patients and a search for new prognostic markers . Indian J Pathol Microbiol. 2013; 56(3): 216-20.

8. Dybko J, Jaźwiec B, Haus O, Urbaniak-Kujda D, Kapelko-Słowik K, Wróbel T, et al. The Hasford score may predict molecular response in chronic myeloid leukemia patients: a single institution experience. Dis Markers. 2016; 2016: 7531472. doi: 10.1155/2016/7531472.

9. Marin D, Bazeos A, Mahon FX. Adherence is the critical factor for achieving molecular responses in chronic myeloid leukemia patients who achieve complete cytogenetic responses on imatinib. J Clin Oncol. 2010; 28(14): 2381-8.

10. Reksodiputro AH. Clinical characteristic and hematologic response to imatinib in patients with chronic phase myeloid leukemia at Cipto Mangunkusumo Hospital. Acta Med Indones. 2010; 42(1): 2-5.

11. Instituti i Shëndetit Publik [Internet]. Roko D, Babameto-Laku A, Mokini V, Cikuli M: Hematologic and cytogenetic responses of Imatinib Mesylate and significance of Sokal score in chronic myeloid leukemia patients [updated 2013 Oct 9; cited 2018 Dec 31]. Available from: http://www.ishp.gov.al/hematologic-andcytogenetic-responses-of-imatinib-mesylate-and-significance-ofsokal-score-in-chronic-myeloid-leukemia-patients/.

12. Ashariati A, Ugroseno YB. Profile of BCR ABL transcript level based on Sokal prognostic score in chronic myeloid leukemia patients treated with imatinib. Acta Med Indones. 2013: 45(2): 10713.

13. Sawyers CL. Imatinib induces hematologic and cytogenetic responses in patientswith chronic myelogenous leukemia in myeloid blast crisis: results of a phase II study. Blood. 2002; 99(10): 3530-9. 
14. Kantarijan HM, Cortes J. Chronic myeloid leukemia. In: Dennis LK, Stephen LH, Dahn LL editors. Harrison Hematology and Oncology. 3rd ed.New York: Mc Graw Hill; 2017. p.181.

15. Liesveld JL, Licthman MA. Chronic myelogenous leukemia an related disorders. In: Kaushanky K, Licthman MA, editors. William Manual of Hematology. 9th ed. New York: Mc Graw hill; 2016. p. 1437.

16. Bilen Y, Erdem F. Hematologic, cytogenetic, and molecular responses to imatinib therapy for chronic myeloid leukemia:a single-center experience in Turkey. Turk J Med Sci. 2012; 42(1): 31-8.

17. Usman M, Syed NN, Kakepoto GN, Adil SN, Khurshid M. Chronic phase chronic myeloid leukemia: response of imatinib mesylate and significance of Sokal score, age and disease duration in predicting the hematological and cytogenetic response. J Assoc Physicians India. 2007; 55: 103-7.

18. Elbedewy TA. The utility and applicability of chronic myeloid leukemia scoring systems for predicting the prognosis of Egyptian patients on Imatinib: retrospective study. J Leuk 2016; 4: 210. doi:10.4172/2329-6917.1000210
19. Xia L, Qian W, Yang M, Li Q, Liu F, Xie Y. Comparison of the utility and applicability of the Sokal, Hasford, and EUTOS scores in a population of Chinese patients with chronic-phase chronic myeloid leukemia undergoing imatinib therapy. Onco Targets Ther. 2015; 8: 2485-92.

20. Yamamoto E, Fujisawa S, Hagihara M, Tanaka M, Fujimaki K, Kishimoto $\mathrm{K}$, et al. European treatment and outcome study score does not predict imatinib treatment response and outcome in chronic myeloid leukemia patients. Cancer Sci. 2014; 105(1): 105-9.

21. Kuntegowdanahalli LC, Kanakasetty GB, Thanky AH, Dasappa L, Jacob LA, Mallekavu SB, et al. Prognostic and predictive implications of Sokal, Euro and Eutos scores in chronic myeloid leukaemia in the imatinib era-experience from tertiary oncology centre in Southern India. Ecancermedicalscience. 2016;10: 679. doi: 10.3332/ecancer.2016.679.

22. Castagnetti F, Palandri F, Amabile M, Testoni N, Luatti S, Soverini S, et al. Results of high-dose imatinib mesylate in intermediate Sokal risk chronic myeloid leukemia patients in early chronic phase: a phase 2 trial of the GIMEMA CML Working Party. Blood. 2009; 113(15): 3428-34. 